\title{
Evaluación de la Satisfacción Laboral en Docentes de Nivel Básico
}

\section{EVALUATION OF WORK SATISFACTION IN PRIMARY SCHOOL TEACHERS}

\author{
Daniel Páramo', Corina Flores Hernández², Luz Verónica Díaz De León² \\ 1. Universidad de Guanajuato, León, Guanajuato, México \\ 2. Universidad DeLaSalle Bajío, León, Guanajuato, México
}

\section{RESUMEN}

El objetivo fue evaluar la satisfacción laboral en docentes de nivel básico utilizando la Escala General de Satisfacción Laboral de Warr, Cook y Wall de 1979. Esta evaluación además dio luz acerca de la escala misma: su confiabilidad y su correlación interna. Se seleccionaron al azar 27 docentes y se les aplicó el instrumento consistente en 15 items con respuesta en escala tipo Likert. Entre los resultados más notables destacó el moderado nivel de satisfacción laboral que mostraron. Se identificaron tres items más elevados, dos de ellos estimadores de la satisfacción extrínseca. La medición de la confiabilidad fue buena para el instrumento en su totalidad con una alfa de Cronbach de 0,879. La correlación interna entre subescalas fue muy buena, del 96\%. Se concluye que, aunque la población estudiada no mostró alta satisfacción por el trabajo, la escala es adecuada para su uso en profesores en nuestro contexto.

(Páramo D, Flores C, Díaz De León L, 2016. Evaluación de la Satisfacción Laboral en Docentes de Nivel Básico. Cienc Trab. Sep-Dic; 18 [57]: 173-176).

Palabras clave: SATISFACCIÓN EN EL TRABAJO, CULTURA ORGANIZACIONAL, DOCENTES.

\section{ABSTRACT}

The objective was to evaluate job satisfaction in teachers at the entry level using the General Work Satisfaction Scale of Warr, Cook and Wall of 1979. This evaluation also gave the scale itself: its reliability and its internal correlation. Twenty-seven teachers were randomly selected and the instrument consisted of 15 items with a Likert-type response. Among the most notable results was the moderate level of job satisfaction they showed. Three higher items were identified, two of them estimators of extrinsic satisfaction. The reliability measurement was good for the instrument as a whole with a Cronbach's alpha of 0.879 . The internal correlation between subscales was very good, 96\%. It is concluded that, although the studied population did not show high satisfaction for work, the scale is suitable for use in teachers in our context.

Keywords: SATISFACTION AT WORK, ORGANIZATIONAL CULTURE, TEACHERS.

\section{INTRODUCCIÓN}

El comportamiento de los trabajadores en el marco de su actividad laboral depende de múltiples factores. De acuerdo con Robbins ${ }^{1}$, las variables más estudiadas en este contexto son: compromiso con el trabajo, satisfacción laboral y compromiso organizacional. La satisfacción en el trabajo forma parte de la actitud general de un individuo hacia su empleo. Se relaciona directamente con la satisfacción del cliente o usuario que, por lo tanto, exige el compromiso del personal.

Entonces es importante distinguir entre la satisfacción del usuario y la del trabajador. La primera es una experiencia cognoscitiva

\section{Correspondencia / Correspondence:}

Dra. Corina Flores Hernández

Avda. Universidad no. 602 Colonia Lomas del Campestre

1.. Sección. C.P. 37150

Tel.: 52+477 $7108500 /$

e-mail: coria999@yahoo.es

Recibido: 24 de Julio de 2016 / Aceptado 02 de Diciembre de 2016 que deriva de la comparación entre las expectativas y la realidad percibida, ya sea que se refiera al proceso educativo o a los resultados finales, tal y como ocurre en el ámbito de los servicios de salud. ${ }^{2}$ Además de las expectativas intervienen valores morales, culturales, así como necesidades personales y circunstancias de la misma organización. Esto hace que la experiencia de satisfacción pueda ser diferente para distintas personas y para la misma persona en distintas circunstancias.

En el extremo contrario se ubica la satisfacción del empleado. Es el conjunto de actitudes ante el trabajo, es decir, conjunta pensamientos y sentimientos. Locke la definió como el "estado emocional positivo o placentero de la percepción subjetiva de las experiencias laborales del sujeto". Depende de circunstancias del trabajo y características personales, necesidades, expectativas, el entorno cultural, etcétera. A su vez la satisfacción en el trabajo permite conocer el grado de motivación y compromiso que tienen los trabajadores hacia la institución y su trabajo cotidiano. También se ha relacionado con la salud física y psíquica ${ }^{3}$, y con la productividad. ${ }^{4}$

Una de las teorías más influyentes de la satisfacción laboral es la formulada por Herzberg en $1959 .{ }^{5}$ Él postuló la existencia de dos grupos o clases de factores: extrínsecos e intrínsecos. Los primeros se refieren a las condiciones concretas de trabajo como, por ejemplo, el salario, políticas de empresa, ambiente, la seguridad, entre otros. Según este modelo bifactorial los factores extrínsecos 
deben estar presentes en una medida suficiente para evitar la insatisfacción laboral. Sin embargo, estar de acuerdo con el salario recibido o trabajar en un ambiente atractivo, por mencionar dos ejemplos, no generan necesaria y automáticamente una alta satisfacción.

La forma de medir los indicadores de satisfacción es a través de aplicar cuestionarios que exploren dimensiones de estudio como: 1) reconocimiento a la labor, 2) problemáticas laborales, 3) condiciones de trabajo, 4) área física. En el ámbito escolar el cliente no siempre cuenta con la libertad de cesar la relación con el trabajador y esto aumenta la relevancia de estudiar esta variable en docentes. Por tanto, el objetivo fue evaluar la satisfacción laboral en docentes de nivel básico y analizar sus componentes intrínsecos y extrínsecos, así como la relación entre ambos.

\section{MATERIAL Y MÉTODOS}

Estudio de tipo descriptivo utilizando la escala general de satisfacción laboral (Overall Job Satisfaction Scale) de Warr, Cook y Wall que recientemente ha sido puesta a prueba en población hispanoparlante. ${ }^{6}$ Se seleccionaron al azar 27 docentes de una institución educativa de nivel básico $\mathrm{y}$, previo consentimiento informado, se les aplicó el instrumento. En apego a las sugerencias de los autores se aplicó guardando el anonimato de los respondientes para que de esta manera esté asegurada la validez de las respuestas.

La escala utilizada contiene 15 items. Fue elaborada con base en la teoría bifactorial de la satisfacción laboral y pensada para ser utilizada en diversos ámbitos ocupacionales independientemente de la formación académica del trabajador. Está conformada por dos subescalas: 1) Subescala de factores intrínsecos: aborda aspectos como el reconocimiento obtenido por el trabajo, responsabilidad, promoción, aspectos relativos al contenido de la tarea, entre otros. Esta sección se compone por los siete ítems de número par; 2) Subescala de factores extrínsecos: indaga sobre la satisfacción del trabajador con relación a aspectos de la organización del trabajo como el horario, la remuneración, las condiciones físicas del trabajo, etcétera. Esta escala la constituyen ocho items impares $(1,3,5,7,9,11,13$ y 15). Los rangos de respuesta asignan un valor de 1 a la respuesta "muy insatisfecho" y correlativamente aumentan hasta asignar un valor a 7 a "muy satisfecho".

El análisis estadístico se llevó a cabo mediante el programa SPSS v18 con el que primeramente se hizo un análisis univariado de las variables sociodemográficas como el sexo y la edad, así como también se utilizó la prueba Alfa de Cronbach. Los valores para satisfacción intrinseca oscilaron entre 7 y 49 y para la extrínseca entre 8 y 56. Para la satisfacción total la escala osciló entre 15 y 105 puntos. Una vez analizadas las subescalas se procedió a valorar estadísticamente la correlación entre la satisfacción general y la satisfacción intrínseca y extrínseca.

\section{RESULTADOS}

Participaron 27 profesores cuyas edades se distribuyeron entre los 22 y los 58 años con una media de 35,64 \pm 12.5 años. Su formación fue de licenciatura para el 77,8\% del total, 18,5\% tienen especialidad y el 3.7\% tienen maestría. El 77,8\% refirieron ser solteros y el 22,2\%, casados. La distribución por jornada laboral fue de 60\% para el turno matutino y el restante $40 \%$ vespertino.

Tabla 1.

Resultados por ítem de la evaluación de Satisfacción Laboral.

\begin{tabular}{|c|c|c|c|c|c|c|c|}
\hline ITEM & $\begin{array}{c}\text { Muy } \\
\text { insatisfecho } \\
N(\%)\end{array}$ & $\begin{array}{l}\text { Insatisfecho } \\
\text { N(\%) }\end{array}$ & $\begin{array}{l}\text { Moderadamente } \\
\text { insatisfecho } \\
N(\%)\end{array}$ & $\begin{array}{c}\text { Ni insatisfecho } \\
\text { Ni satisfecho } \\
\mathrm{N}(\%)\end{array}$ & $\begin{array}{c}\text { Moderadamente } \\
\text { satisfecho } \\
N(\%)\end{array}$ & $\begin{array}{c}\text { Satisfecho } \\
N(\%)\end{array}$ & $\begin{array}{l}\text { Muy } \\
\text { satisfecho } \\
N(\%)\end{array}$ \\
\hline 1. Condiciones físicas del trabajo & $5(18,5)$ & $7(25,9)$ & $5(18,5)$ & $1(3,7)$ & $5(18,5)$ & $3(11,1)$ & $1(3,7)$ \\
\hline 3. Compañeros de trabajo & $1(3,7)$ & $1(3,7)$ & $3(11,1)$ & $2(7,4)$ & $4(4,8)$ & $11(40,7)$ & $5(18,5)$ \\
\hline $\begin{array}{l}\text { 4. Reconocimiento que obtienes } \\
\text { por el trabajo bien hecho }\end{array}$ & $4(14,8)$ & $5(18,5)$ & $4(14,8)$ & $3(11,1)$ & $7(25,9)$ & $3(11,1)$ & $1(3,7)$ \\
\hline $\begin{array}{l}\text { 6. Responsabjlidad que se te } \\
\text { ha asignado }\end{array}$ & $4(14,8)$ & $2(7,4)$ & $3(11,1)$ & $2(7,4)$ & $5(18,5)$ & $6(22,2)$ & $5(18,5)$ \\
\hline 7. Tu salario & $6(22,2)$ & $5(18,5)$ & $4(14,8)$ & $0(0)$ & $3(11,1)$ & $5(18,5)$ & $4(14,8)$ \\
\hline $\begin{array}{l}\text { 8. Posibilidad de usar } \\
\text { tus capacidades }\end{array}$ & $6(22,2)$ & $4(14,8)$ & $1(3,7)$ & $4(14,8)$ & $4(14,8)$ & $4(14,8)$ & $6(22,2)$ \\
\hline $\begin{array}{l}\text { 9. Relaciones entre dirección y } \\
\text { trabajadores en tu empresa }\end{array}$ & $8(29,6)$ & $2(7,4)$ & $4(14,8)$ & $3(11,1)$ & $4(14,8)$ & $3(11,1)$ & $3(11,1)$ \\
\hline $\begin{array}{l}\text { 12. Atención que se presta a } \\
\text { as sugerencias que haces }\end{array}$ & $8(29,6)$ & $2(7,4)$ & $4(14,8)$ & $4(14,8)$ & $4(14,8)$ & $1(3,7)$ & $4(14,8)$ \\
\hline 13. Tu horario de trabajo & $0(0)$ & $0(0)$ & $0(0)$ & $1(3,7)$ & $5(18,5)$ & $12(44,4)$ & $9(33,3)$ \\
\hline $\begin{array}{l}\text { 14. La variedad de tareas que } \\
\text { realizas en tú trabajo }\end{array}$ & $5(18,5)$ & $0(0)$ & $0(0)$ & $0(0)$ & $4(14,8)$ & $7(25,9)$ & $11(40,7)$ \\
\hline 15. Tu estabilidad en el empleo & $0(0)$ & $12(44,4)$ & $12(44,4)$ & $0(0)$ & $0(0)$ & $0(0)$ & $3(11,1)$ \\
\hline
\end{tabular}


Tabla 2.

Media, desviación estándar y valor Alfa de la evaluación de la satisfacción laboral y sus subescalas.

$\begin{array}{lcc}\text { Escala } & \text { Media } \pm \mathrm{DE} & \text { Alfa de Cronbach } \\ \text { Satisfacción total } & 64,07 \pm 22,9 & 0,879 \\ \text { Satisfacción intrínseca } & 32,19 \pm 8,3 & 0,670 \\ \text { Satisfacción extrínseca } & 39,11 \pm 9,1 & 0,658\end{array}$

En la evaluación realizada la satisfacción laboral resultó ser moderada. El puntaje promedio fue de 64 en una escala que va del 15 a 105 puntos máximos posibles, esto quiere decir que los resultados se ubican en el decil 60. El componente más sólido fue el de la satisfacción extrínseca con un resultado global promedio de 39 de un máximo de 56 puntos, lo que representa una satisfacción de casi el 70\%. Los factores intrínsecos alcanzaron a cubrir 65\% del rango de la escala. Ante estos resultados se hace necesario un análisis por ítem, ya que los puntajes resultaron estar muy polarizados con ítems muy altos y otros muy bajos.

Los resultados por item se reproducen en la Tabla 1. En la referida Tabla se puede observar que en general los profesores mostraron un valor de satisfacción levemente más elevada con relación a los factores externos. En términos generales, los puntajes estuvieron apuntalados por tres items principales: los compañeros de trabajo, los horarios y la variedad de tareas. Los dos primeros son de tipo extrínseco. Paradójicamente, también factores extrínsecos destacaron por tener los puntajes más bajos, sobre todo los items referidos a: las condiciones físicas, salario, la relación entre el nivel directivo y los trabajadores, así como en la estabilidad percibida en el empleo. Los factores intrínsecos más negativos fueron los referidos a la falta de libertad para elegir los métodos de trabajo y la atención que se le presta a las sugerencias hechas por el docente.

Los estadísticos descriptivos se refieren en la Tabla 2, donde también se reportan los valores de la prueba Alfa de Cronbach encontrados. Destaca que la prueba Alfa fue buena para la escala general, y apenas suficiente para las dos subescalas.

En cuanto a la correlación entre la satisfacción total y la intrínseca, se encontró existe una correlación interna de Pearson del 96,0\% entre ambas variables, siendo estadísticamente significativa $(\mathrm{P}<0,001)$ (Figura 1). De modo casi idéntico, la relación entre satisfacción laboral general y extrínseca mostró un coeficiente de correlación de 0,95 y una $\mathrm{P} \leq 0,000$.

Figura 1.

Correlación entre satisfacción laboral general y satisfacción intrínseca.

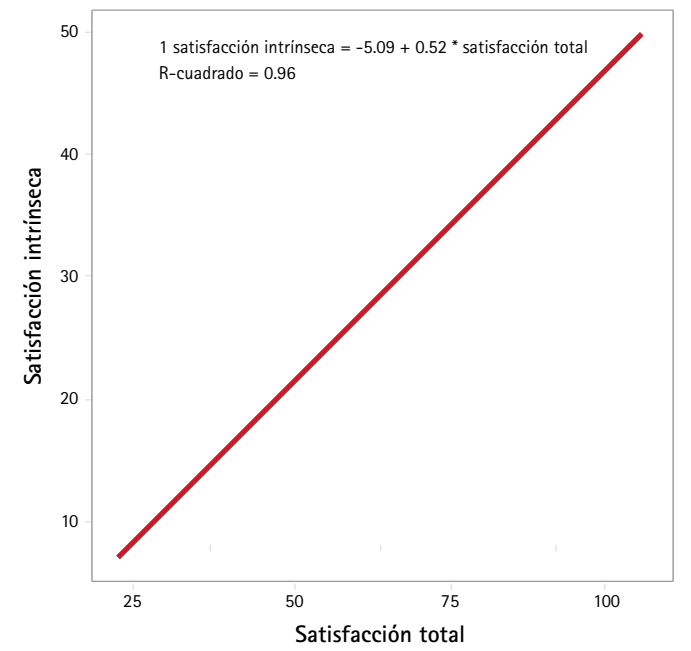

Figura 2.

Correlación entre satisfacción laboral general y satisfacción extrínseca.

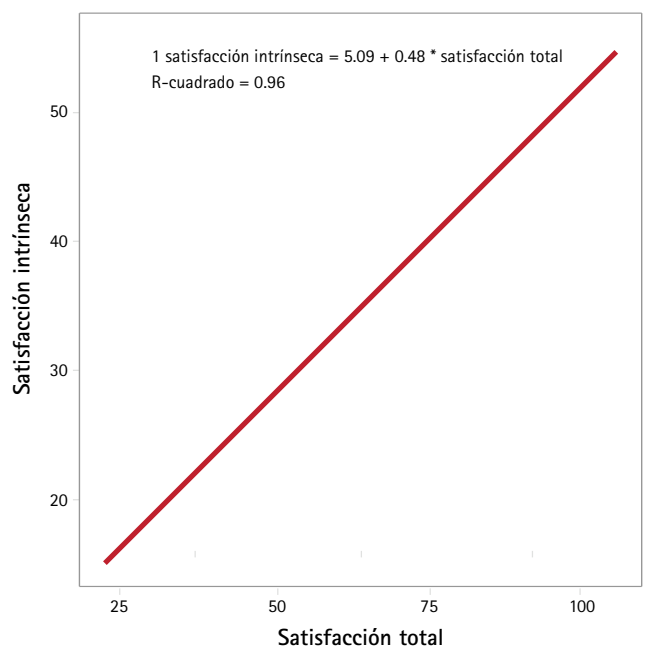

\section{CONCLUSIONES}

En cualquier organización es importante evaluar la satisfacción de los empleados y las instituciones educativas no son la excepción. Para muchos autores la satisfacción en el trabajo es un motivo en sí mismo; para otros es expresión de una necesidad que puede o no ser satisfecha. En cualquier caso, el grado de satisfacción del trabajador tendrá repercusiones en el proceso educativo. En el presente estudio se documentó el grado moderado de satisfacción, principalmente debida a los factores extrínsecos. Sin embargo, el análisis de los ítems individuales, así como el de la relación entre las subescalas, revelaron otros aspectos relevantes.

El análisis pormenorizado de los puntajes revela que ninguna de las dos subescalas explica por sí sola la satisfacción, pues ambas mostraron puntuaciones elevadas y bajas. No obstante, predominaron las puntuaciones elevadas en los factores extrínsecos. Esto recalca la importancia de este tipo de evaluaciones en cada institución, ya que los resultados en poblaciones dadas no pueden ser generalizados directamente sino hasta después de un cuidadoso análisis de las circunstancias objetivas de las organizaciones, y de la adecuada comprensión de su personal.

Aunque desde una perspectiva teórica diferente, recientemente se ha comprobado el importante papel de las necesidades psicológicas en el profesorado. ${ }^{7}$ Estas necesidades psicológicas son entendidas aquí como factores intrínsecos y conforman el abanico de estrategias con el que las personas hacen frente y se adaptan a las condiciones de trabajo.

Por otra parte, el análisis de las subescalas demostró que consideradas de manera independiente, ninguna de las dos obtuvo una confiabilidad elevada. Sin embargo, combinadas es muy buena con una alfa de 0,879 . Esto confirma la solidez del instrumento como una unidad y, por lo tanto, se recomienda para aplicarse en otros espacios laborales.

\section{Agradecimientos}

Agradecemos sinceramente a los docentes por su apoyo para proyecto. 


\section{REFERENCIAS}

1. Robbins SP. Comportamiento Organizacional. 8a ed. México: Prentice Hall; 1998.

2. Donabedian A. Evaluating the quality of medical care. Milbank 0. 2005; 83(4):691-729.

3. Zurlo MC, Pes D, Capasso R. Personality characteristics, job stressors, and job satisfaction: main and interaction effects on psychological and physical health conditions of italian school teachers. Psychol Rep [on line]. 2016 [cited feb 2016]:1-12. Available from: http://prx.sagepub.com/ content/early/2016/07/04/0033294116656818.full.pdf

4. Brown A, Kitchell M, O'Neill T, Lockliear J, Vosler A, Kubek D, Dale L. Identifying meaning and perceived level of satisfaction within the context of work. Work. 2001; 16(3):219-226.
5. Garcia D. Satisfacción Laboral. Una aproximación teórica. Contribuciones a las Ciencias Sociales-EUMEDNET [en línea]. jul 2010 [consultado feb 2016]. Disponible en: www.eumed.net/rev/cccss/09/dgv.htm

6. Boluarte A. Propiedades psicométricas de la Escala de satisfacción laboral de Warr, Cook y Wall. Rev Med Hered. 2014; 25:80-84.

7. Betoret F. Teacher Psychological Needs, Locus of Control and Engagement. Span J Psychol [on line] 2013 [cited apr 2016];16: E29 Available from: http://journals.cambridge.org/action/displayAbstract?fromPage=online\&ai $d=8937047 \&$ fileld $=$ S1138741613000516 